https://doi.org/10.48009/2_iis_2007_478-483

\title{
INTERNET EXPLORER AND FIREFOX: WEB BROWSER FEATURES COMPARISION AND THEIR FUTURE
}

\author{
Siwat Saibua, Texas A\&M University-Kingsville, siwat.saibua@tamuk.edu \\ Joon-Yeoul Oh, Texas A\&M University-Kingsville, joon-yeoul.oh@tamuk.edu \\ Richard A. Aukerman, Texas A\&M University-Kingsville, richard.aukerman@tamuk.edu
}

\begin{abstract}
Internet technology is one of the utmost inventions of our era and has contributed significantly in distributing and collecting data and information. Effectiveness and efficiency of the process depends on the performance of the web browser. Internet Explorer is the leader of the competitive browser market with Mozzilla Fox as its strongest rival, which has been and is gaining a substantial level of popularity among internet users. Choosing the superlative web browser is a difficult task due to the considerably large selection of browser programs and lack of tangible comparison data. This paper describes and compares vital features of Internet Explorer and Mozzilla Firefox, which represent over $90 \%$ of the browser market. The performance of each browser is evaluated based on the general features, operating system support, browser features, protocol support and language support. The paper is concluded with concrete remarks based on the comparison.
\end{abstract}

Keywords: Information Technology (IT), Web Browser, Software Evaluation, Internet Protocol, Search Engines, Mozilla Firefox, Internet Explorer,

\section{INTRODUCTION}

A web browser is a software program that allows accessing of information on the internet. Followed by the WorldWideWeb software that was written by Tim Berners-Lee and released to the public in 1991, numerous web browsers have been developed and released to the market. As a result, the Internet users have multiple web browsers to choose from which presents the unavoidable question of "Which is the best browser in the market?" Regardless of web browser developers' efforts to remain competitive in the market, the life of web browsers is rather short. Unlike most browsers, including Netscape, Internet Explorer (IE) that was developed by Microsoft in 1995 has remained most popular among internet users.
Next to Netscape, which was introduced to the market in 1998, Mozilla Firefox was released in 2004 to compete with IE. The software codes of Firefox are in the open source format, and any software developers around the world can put their own ideas into this browser. As a result, Firefox's performance effectiveness and efficiency improved every day and gained popularity rapidly.

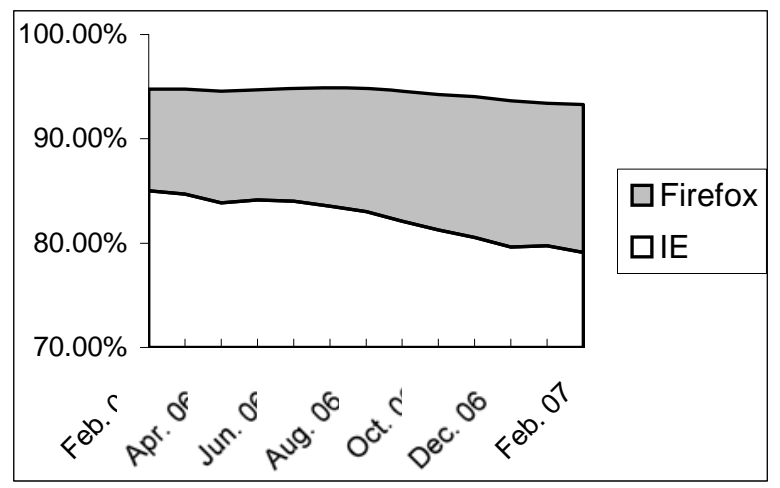

Figure 1: Market shares between IE and Firefox

According to netapplications.com, headquartered in Aliso Viejo, CA, the market share of Internet Explorer has declined to $79.09 \%$ while that of Firefox has been increased to $14.18 \%$ of the global usage share by February 2007 [4]. Opera, Apple's Safari, Netscape and others make up the rest. Figure 1 shows that Firefox's growth of $45.44 \%$ while Internet Explorer's decreased its market share by $6.99 \%$ within a year. In Europe, IE's market share is even less than in the US. For example, in Germany, Firefox's market share was $39.02 \%$ while IE's was $55.99 \%$ as of July 2006, which is about a double of global usage share rate. By January 2007, Germany's Firefox users dropped to $25.66 \%$ with the usage rate for IE increasing to $69.47 \%$ [10]. The global market share of Firefox, on January 2007, had decreased to $13.67 \%$ from $14.00 \%$ on December 2006 [4]. However, the difference is not significant enough to impact the global usage share of FireFox, and in February 2007, Firefox's market share started to 
increase again. Even with FireFox's gradual increase in global market share, many predict that the war of web browsers will remain strong due to the growing demands of over 1.6 million internet users, or $16.6 \%$ of the global population, for enhancement of browser performance in terms of power, speed, security and more.

This paper discusses and compares the various features of two leading web browser competitors, Firefox and IE. First of all, a brief history of IE and Firefox is discussed in the next section. This is followed by a comparison of various features such as general features, operating system support, browser features, protocol support, and language support, of both web browsers. Finally, conclusions and comments are stated.

\section{HISTORY OF INTERNET EXPLORER AND MOZILLA FIREFOX}

\section{Internet Explorer History}

The origin of Internet Explorer is Spyglass Mosaic, the first popular web browser, developed at the National Center for Supercomputing Applications (NCSA) in late 1992. However, neither IE version 1.0, released in July 1995, nor IE version 2.0, released in November 1995, was particularly popular until IE version 3.0 with cascading style sheets (CSS) support was released in 1996. The version 3.0 also provided more security; however, researchers and hackers found security and privacy vulnerabilities several months after its release.

In September 1997, IE version 4.0 was released with supporting Group Policy which is an infrastructure used to deliver and apply one or more desired configurations or policy settings to a set of targeted users and computers within an Active Directory environment for centralized management [7]. Internet Explorer 5.0 was another significant release in September 1999 with bi-directional text support that allows writing both left-to-right and right-to-left directions, ruby characters. The Dynamic HTML (DHTML) of IE 5.5, released in 2000, allowed more personalized web applications, such as interactive and animated web sites using a combination of HTML, JavaScript, and CSS. This technology contributed heavily to the rapid growth of Ecommerce [12].

In 2001, Internet Explorer version 6 was released with Windows XP providing more private, reliable, and flexible technology than previous versions using the Platform for Privacy Preferences (P3P). The P3P was developed by the World Wide Web Consortium (W3C) to satisfy the internet users' privacy and security demands, such as protecting against tracking users' web surfing. Including the P3P, Internet Explorer Administration Kit (IEAK), Media bar, Windows Messenger integration, and automatic image resizing were introduced in this version.

The final public version of Internet Explorer 7 was released in October 2006 including new technologies, such as built-in tabbed browsing, antiphishing technology, Really Simple Syndication (RSS) reader, and a redesigned Favorites Center. However, IE 7 is limited to the users that have Windows XP SP2 or a later version and does not comply with some current Web standards. Two unusual requests, turning off antivirus protection while installing and the rebooting requirement after installation, are also drawbacks of this version. According to cnet.com, the reviewers' rating of IE 7 is 7.5 out of 10 in the editors' review and 4.7 in the users' review while Firefox's is 8.3 and 7.8 respectively in March 2007 [11].

\section{Mozilla Firefox History}

Although Firefox (Fx or FF) is owned by Mozilla Foundation, it is free and open source software and the company's profits are reinvested to Mozilla projects unlike Microsoft. Firefox started under a different name, Phoenix 0.1 released on September 23, 2002. Phoenix 0.1 had a lot to offer such as a download manager, which IE 6 (IE version of that time period) did not contain, a popup blocker and tabbed browsing. A few days after releasing Phoenix 0.1 , the version 0.2 was released on October 10, 2002 with new features, such as sidebar, the ability to add extensions and themes to the browser. After adding new features, such as a default search engine, bug fixing, an improved popup blocker, and improved tool bars for the version $0.3,0.4$ and 0.5 , the name was changed to Thunderbird due to the trademark issues with a company called Phoenix Technologies who produces a BIOS-based browser called Phoenix FirstWare Connect.

However, after releasing Firebird version 0.6, 0.7 and 0.7.1, the name Firebird would have to be changed again to Mozilla Firefox in February 9, 2004 because Firebird was the name of an open source database development project [3]. On November 9, 2004, the Firefox project updated many versions of Fx before the $\mathrm{Fx}$ version 1.0 that supports 31 languages was released. After releasing several 1.x versions, an update was required due to the bug and security fixes of older versions. Mozilla Foundation released its 
first major update to Fx 1.5 on November 29, 2005 and Fx 2.0 on October 2006.

Mozilla is now working on a new version. Two test versions of Fx 3.0, alpha 1 and alpha 2 were released on December 8, 2006 and February 2, 2007, respectively. According to the Mozilla's release roadmap written by Christopher Beard, who has the responsibility for Products and Marketing at the Mozilla Corporation, the final version of Fx 3.0 will be released in November 2007 [1]. Based on alpha releases in Fx 3.0, the largest change of Fx 3.0 is the implementation of Gecko 1.9, a web browser layout engine.

\section{IE VERSUS FX}

There are many web browsers available to the public, but the market share rates for all are very low except for Internet Explorer that has major popularity and Firefox that has gained popularity rather rapidly after its new and improved releases. In this section, the differences between the latest version of IE (i.e. IE 7.0) and Fx (i.e. Fx2.0) are discussed.

\section{General Features Comparison}

Table 1 shows a comparison of the general features of IE and Fx. The layout engine is required to display web content after formatting its contents using the transformation languages, such as CSS and Extensible Stylesheet Language (XSL) [2]. Included in Mozilla Firefox, Gecko layout engine is applied to AOL for Mac OS X, Netscape, etc, while IE uses

Table 1: General features comparison [13]

\begin{tabular}{|l|c|c|}
\hline \multirow{2}{*}{$\begin{array}{l}\text { General } \\
\text { Features }\end{array}$} & \multicolumn{2}{|c|}{ Browser } \\
\cline { 2 - 3 } Creator & Microsoft & $\begin{array}{c}\text { Mozilla } \\
\text { Foundation }\end{array}$ \\
\hline \hline Cost & Free & Free \\
\hline Open Source & No & Yes \\
\hline $\begin{array}{l}\text { Software } \\
\text { license }\end{array}$ & Proprietary & $\begin{array}{c}\text { MPL/GPL/LGPL } \\
\text { tri-license }\end{array}$ \\
\hline $\begin{array}{l}\text { Current } \\
\text { Layout } \\
\text { Engine }\end{array}$ & Trident & Gecko \\
\hline
\end{tabular}

Trident, MS version of a layout engine, is known as the most popular one, and Gecko is chasing Trident's popularity. Unlike the IE's proprietary license structure, Firefox's free and open source policy plays a key role in its rapid growth and active software development efforts.

\section{Operating System Support Comparison}

Microsoft's Internet Explorer has a dedicated operating system, MS Windows series, and acquired over $90 \%$ of market share in March 2007 [5]. Firefox supports most operating systems including Windows. In January 1996, Microsoft released a Mac version of IE to only support the Mac operating system that had a market share of 4.29\% [5]; however, Microsoft ceased further development of the Mac version of IE in December 2005. The latest version of IE supporting Mac OS was IE for Mac 5.2.3. IE 3 or later versions supported Unix OS until IE 5.01 SP1, but stopped the support because of the stability issue. Even though IE is dedicated for Windows OS, IE 7 only supports Windows XP SP2 or later versions.

Table 2: Operating system support [13]

\begin{tabular}{|l|c|c|}
\hline \multirow{2}{*}{$\begin{array}{l}\text { Operating } \\
\text { System Support }\end{array}$} & \multicolumn{2}{|c|}{ Browser } \\
\cline { 2 - 3 } & IE 7 & Fx 2 \\
\hline \hline Windows & Yes (partially) & Yes \\
\hline Mac OS X & No & Yes \\
\hline Mac OS 9 & No & No \\
\hline Linux & No & Yes \\
\hline BSD & No & Yes \\
\hline Unix & Stopped & Yes \\
\hline
\end{tabular}

\section{Browser Features Comparison}

IE users may notice the interface differences between IE 7 and older versions, IE 6 or earlier. The significant improvements of IE 7's new interface include tabbed browsing, integrated searching and news feeds (RSS) support. Compare to IE 7, Fx 2 did not have significant changes from Fx version 1.5 other than the capability of cleaning up tabbed browsing, news feeds support and add-on management. Fx 2's tab updates are generally a step ahead of IE's. For example, the last session can be saved for future use when configuring Firefox while clicking a box every time on IE 7. Firefox also reopens closed tabs via the History menu or by rightclicking an open tab and each Firefox tab has its own closing button that IE 7 also provides. However, 
unlike IE 7, Firefox provides no thumbnail previews of open tabs.

RSS feeds provide effective and efficient ways of accessing or checking the news in real-time and automatically updates from different websites without the users visiting corresponding sites manually. RSS delivers its information as an Extensible Markup Language (XML) file. Though the technology was developed and first used in Fx 1.5, now it is the new support for IE 7, and is also upgraded in Fx 2. Fx 2 makes the raw feed understandable, and offers a range of new subscription options.

Table 3: Browser features comparison [9]

\begin{tabular}{|l|c|c|}
\hline \multirow{2}{*}{ Browser Features } & \multicolumn{2}{|c|}{ Browser } \\
\cline { 2 - 3 } & IE 7 & Fx 2 \\
\hline \hline Tabbed browsing & Yes & Yes \\
\hline Add-ins & Yes & Yes \\
\hline Themes & No & Yes \\
\hline $\begin{array}{l}\text { Built-in search with } \\
\text { multiple engine choice }\end{array}$ & Yes & Yes \\
\hline Pop-up blocker & Yes & Yes \\
\hline Anti-Phishing & Yes & Yes \\
\hline $\begin{array}{l}\text { One click button to add } \\
\text { favorites }\end{array}$ & Yes & No \\
\hline RSS feeds & Yes & Yes \\
\hline Download Manager & No & Yes \\
\hline $\begin{array}{l}\text { Can remember open tabs } \\
\text { for next session }\end{array}$ & Yes & No \\
\hline $\begin{array}{l}\text { Save group of tabs as } \\
\text { bookmark }\end{array}$ & Yes & Yes \\
\hline Thumbnails for tabs & Yes & No \\
\hline BitTorrent client & No & No \\
\hline $\begin{array}{l}\text { Spell-checker for text } \\
\text { boxes }\end{array}$ & No & Yes \\
\hline Download pause & No & Yes \\
\hline
\end{tabular}

Add-ins or Add-ons is also one of the prominent features allowing users to personalize their own web browser. IE 7 with a new add-on manager allows users to enable, disable, and delete the feature. In spite of such a capability, it lacks descriptions of each add-on that makes such a feature problematic. Moreover, to update plug-ins of IE 7, new versions must be checked manually and downloaded. In contrast, $\mathrm{Fx} 2$ built an unambiguous and uncomplicated manager that controls themes and extensions in one window. As in the previous versions, each one has a corresponding description, and allows users to check updates efficiently for every add-on using the Find Updates Button [11].
Both browsers add anti-phishing features to protect against malicious fake sites that attempt to trick users into divulging their information. IE 7 includes site analysis that will try to warn you about a suspicious site even if it is not yet on a blacklist, which is an approach similar to that implemented in the latest security suites. IE 7 initiates an anti-phishing feature by scanning the URL or page content, then sending the addresses of suspicious sites to Microsoft to compare against a blacklist. If a site is on the list, IE will block the page. If the site is a known safe site, the page displays. If the site is unrecognized by the list, it warns the users. In contrast, Fx 2 compares the questionable sites against a locally stored blacklist with an option of choosing to send the visited URLs to Google to compare them against a frequently updated list.

Overall, the IE 7 has many security fixes including a parsing module that identifies and discards dangerous URLs, turning off most ActiveX controls by default, and offering color-coded warnings in the URL bar based on the credibility of the sites. A built-in phishing filter is another new safety enhancement feature that spots malicious pages before they reach the user. The noted security fixes are indispensable and only available for IE 7 that discloses the severity of security vulnerability in the earlier versions including the IE 6. In addition to these major changes, there also have been some minor feature changes such as Spell-checker for Fx 2 and quick page zooms for IE 7.

\section{Protocol Support Comparison}

Table 4 shows a list of protocols supported by both web browsers.

Table 4: Protocol support comparison [13]

\begin{tabular}{|l|c|c|}
\hline \multirow{2}{*}{$\begin{array}{l}\text { Protocol } \\
\text { Support }\end{array}$} & \multicolumn{2}{|c|}{ Browser } \\
\cline { 2 - 3 } HTTP & IE 7 & Fx 2 \\
\hline Email & No & Yes \\
\hline FTP & Yes & Yes \\
\hline NNTP & No & No \\
\hline SSL & Yes & Yes \\
\hline IRC & No & No \\
\hline Gopher & No & Yes \\
\hline IDN & Yes & Yes \\
\hline data:URIs & No & Yes \\
\hline BitTorrent & No & No \\
\hline
\end{tabular}


Note that both browsers do not support email, Network News Transfer Protocol (NNTP) and Internet Relay Chat (IRC) since they have their own supporting mail clients; MS Outlook, Messenger and Windows Live mail desktop for Microsoft and Mozilla Mail and Newsgroups and Mozilla Thunderbird for Mozilla Foundation. Also, both browsers also do not support BitTorrent, a P2P file distribution protocol that distributes a large amount of data widely without the original distributor incurring costs. IE 6 supports Gopher that is a distributed document search and retrieval network protocol, but it was turned off by default $[2,8]$. IE 7, however, does not support the protocol while Fx 2 still supports it.

\section{Language Support Comparison}

Internet Explorer 7 supports 33 languages while Firefox 2 supports 41 languages.

Table 5: Language Support comparison [13]

\begin{tabular}{|l|l|}
\hline \multicolumn{2}{|c|}{ Language Support } \\
\hline \multicolumn{1}{|c|}{ IE 7 } & \multicolumn{1}{c|}{ Fx 2 } \\
\hline \hline Arabic, Brazilian, & Afrikaans, Albanian, \\
Bulgarian, Catalan, & Arabic, Asturian, \\
Chinese, Croatian, & Basque, Bulgarian, \\
Czech, Danish, Dutch, & Catalan, Chinese, \\
English, Estonian, & Czech, Danish, Dutch, \\
Finnish, French, & English, Finnish, \\
German, Greek, & French, Frisian, \\
Hebrew, Hungarian, & Georgian, German, \\
Italian, Japanese, & Greek, Gujarati, \\
Korean, Latvian, & Hebrew, Hungarian, \\
Lithuanian, Norwegian, & Irish, Italian, Japanese, \\
Polish, Portuguese, & Korean, Kurdish, \\
Romanian, Russian, & Lithuanian, \\
Slovak, Slovenian, & Macedonian, \\
Spanish, Swedish, Thai, & Mongolian, Norwegian, \\
Turkish & Polish, Portuguese, \\
& Punjabi, Romanian, \\
& Russian, Slovak, \\
& Slovenian, Spanish, \\
& Swedish, Turkish, \\
& Welsh \\
\hline
\end{tabular}

For the language support, Fx2 is more advanced and diversified than IE 7. Perhaps such advancement is one of the benefits of being a free-open source software permitting users to contribute their own language skills.

\section{CONCLUSIONS AND COMMENTS}

This paper offered a comparison of two leading web browsers, Internet Explore and Firefox, along with their brief histories. Making a decision on determining the more advanced browser between IE and Fx is straightforward based on the various figures in the tables. However, making such a decision is not as objective as it seems when dealing with people. The majority of average users tend to prefer the products they are already familiar with. Unlike the expert users, average users do not detect the significant performance differences between IE 7 and Fx 2. Hence, minor or unapparent flaws in each product are irrelevant when deciding on a browser. Therefore, the futures of IE and Fx are uncertain and this competition will continue at least for several years even with Fx 2's rapid growth in the market and frequent criticism of Microsoft for copying FireFox's dominant features such as user interface and tabbed browsing windows.

In order to survive the war of web browsers, the four fundamental functional requirements, which are security, compatibility, velocity and stability, must be addressed and satisfied. Both IE 7 and Fx 2 are new to the market; hence, their security vulnerabilities are yet to be discovered by users, especially expert users, and hackers. The security vulnerability of IE 7 is probably greater than Fx 2 since illegal hackers tend to target the software industry leaders such as Microsoft. Along with security, a web browser must satisfy compatibility concerns. With the significant growth in internet and/or software industries, supporting protocols and technologies of web browsers must keep up with the industry needs.

In conclusion, it is rather difficult to objectively decide on the winner of web browser competition at this stage. In order to remain strong in the market, developers must further identify and meet the requirements of internet customers. After all, competitiveness of the web browser market is a good indication of companies' level of effort in supporting customers.

\section{REFERENCES}

1. Beard, C. (2006). Firefox release roadmap, Retrieved February 27, 2007, from http://wiki.mozilla.org/index.php?title=ReleaseR oadmap\&oldid=40597

2. Carr, H. H., \& Snyder, A. C. (2007). Data Communications and Network Security. New York, NY: McGraw-Hill. 
3. flexbeta.net (2005). The History of Mozilla Firefox: From Phoenix, to Firebird, to Firefox, Retrieved February 28, 2007, http://www.flexbeta.net/main/printarticle.php?id $=89$

4. Market Share by Net applications (2007). Top Browser Market Share Trend for March 2006 to February 2007. Retrieved March 5, 2007, from http://marketshare.hitslink.com/report.aspx?qpri $\mathrm{d}=3$.

5. Market Share by Net applications (2007). Operating System Market Share for February 2007. Retrieved March 5, 2007, http://marketshare.hitslink.com/report.aspx?qpri $\mathrm{d}=2$

6. Microsoft Corporation Press (2003). Windows History: Internet Explorer History, Retrieved March 3, 2007, from http://www.microsoft.com/windows/WinHistory IE.mspx

7. Microsoft Corporation Press (2006). Group Policy Frequently Asked Questions (FAQ), Retrieved March 6, 2007, from http://technet2.microsoft.com/windowsserver/en/ technologies/featured/gp/faq.mspx
8. Microsoft Corporation Press (2006). Release notes for Internet Explorer 7, Retrieved March 1, 2007, from http://msdn2.microsoft.com/enus/ie/aa740486.aspx

9. Muchmore, W. M (2006). Which New Browser Is Best: Firefox 2, Internet Explorer 7, or Opera 9? Retrieved February 27, 2007, http://www.extremetech.com/article2/0,1697,199 0850,00.asp

10. OneStat Press (2007). Microsoft's Internet Explorer global usage share is 85.81 percent according to OneStat.com, Retrieved March 6, 2007, http://www.onestat.com/html/aboutus_pressbox5 0-microsoft-internet-explorer-7-usage.html

11. Robert Vamosi (2006). Cnet editors' review, Retrieved March 6, 2007, from http://reviews.cnet.com/Internet_Explorer_7/450 5-3514_7-32111537.html

12. Stamper, A. D., \& Case, L. T. (2003). Business Data Communications ( $6^{\text {th }}$ ed.). Upper Saddle River, New Jersey: Prentice Hall.

13. Wikipedia.com (2007). Comparison of web browsers, Retrieved March 5, 2007, from http://en.wikipedia.org/wiki/Comparison_of_we b_browsers 